\title{
Aligning Software Maintenance to the Offshore Reality
}

\author{
Christian Seybold \\ Zühlke Engineering $A G$ \\ Zürich-Schlieren, Switzerland \\ cseybold@gmx.ch
}

\author{
Rudolf K. Keller ${ }^{*}$ \\ Zühlke Engineering $A G$ \\ Zürich-Schlieren, Switzerland \\ keller@pmod.com
}

\begin{abstract}
With an ever-increasing amount of software in place, the importance and ubiquity of software maintenance is continuously growing. Another more recent trend is to "offshore" such work to low-wage countries with still untapped engineering resources. However, software maintenance typically requires intensive customer contact, short iteration cycles, and fast response times which is counter to the communication delays, requirements misinterpretations and indirect responsibilities often found in offshore cooperations.

This paper reports on a software maintenance project in the power plant engineering sector. From the outset, the project included, in line with corporate policy, heavy offshore involvement. This 'offshore reality' confronted the stakeholders with various problems, including lack of quality, slipped deadlines, and communication overhead. A number of measures were introduced to mitigate the problems. Finally, a project setup could be found satisfying both the characteristics of software maintenance projects and the peculiarities of offshore cooperations. In the paper, we describe and analyze the course of this project, and present a list of lessons learned and best practices for this uncommon, yet increasingly important project setup.
\end{abstract}

\section{Introduction}

Businesses are spending large amounts of money on offshore and near-shore outsourcing, but many of the offshore projects have failed because of poor planning and wrong setups [7]. Sometimes, software maintenance projects are quoted as project types that are fit for offshore development. However, software maintenance typically requires intensive customer contact, short iteration cycles, and fast response times which is counter to the communication delays, requirements misinterpretations and indirect responsibilities often found in offshore cooperations [9, 11].

In our recent work at Zühlke Engineering AG, henceforth "Zühlke", we were involved in a major software maintenance project in the power plant industry. The management of the industrial partner had long before decided to pursue a rigorous offshore strategy, that is, the requirement to develop and maintain mostly offshore was a given and unquestionable.

This "offshore reality", coupled with a host of problems not untypical for a project that had suffered from underfunding for some time, raised a host of problems. We were poised to apply best software engineering practices, yet were challenged with the peculiarities of the offshore context. In the course of our 16 months involvement in the project, we came up with a number of measures to mitigate these challenges. Anecdotal evidence and some quantitative data suggest that these measures indeed had a positive impact and contributed to the success of the project. We think that the measures apply to similar offshore maintenance projects, and to a large extent, also to maintenance projects in general.

In this paper, we first provide an overview of the project at hand. Then, we detail the problems and challenges we were facing at the outset of the project. The centerpiece of the paper is the discussion of the measures taken to bring and keep the project on course. This discussion is followed by an analysis of the impact of the measures. Furthermore, a list of lessons learned is presented. Finally, in the conclusion, we briefly discuss the usefulness of applying offshoring in maintenance projects, and reflect on the factors that made the project under consideration a successful one.

\footnotetext{
* The second author carried out part of this work as an Adjunct Professor at Université de Montréal, Canada.
} 


\section{Project Background}

The project under study is being carried out by a customer in the power engineering sector. From the outset, Zühlke has played a major role in its implementation. The objective of the project is the development of a number of business applications for information management. These applications (about ten in total, of which four of them are major ones) are organized in a three-tier architecture consisting of a presentation layer as web application, a layer for the business logic and a layer for data handling. The project started at the beginning of 2001 and is still evolving. The course of the project can be divided into four different phases (see Figure 1):

1. From May'01 to Dec'04, these applications were developed from scratch with heavy involvement of Zühlke (5 engineers on the average). The focus was on high-quality forward development up to live release and handover to the end users.

2. From Jan'05 to Jul'05, the project was mainly dormant for lack of resources (both internal and external). Maintenance was limited to the implementation of minor change requests and bug fixes. Only the most important issues could be handled to keep the applications running.

3. Starting in Aug'05 until today, our customer decided to obtain support by an offshore partner in India. The size of the offshore team has increased from one person in the beginning to six persons today. After the previous, rather quiet phase, there was a high demand for the development of new features and the implementation of change requests. Development was back at the center of attention, yet hardly any activities concerning quality were performed. From Jan'05 to Jun'06, the applications continuously decayed. Arguably, the major problems were related to the new project setup involving distributed development with the offshore partner.

4. Due to the growing number of problems concerning quality and release management, support from Zühlke was ordered again. Thus, as of Jul'06, the customer's onshore team consisting of one developer and a part-time project leader was complemented with a senior engineer from Zühlke, incidentally the first author of this paper (with the second author taking the role of mandate owner). The 16 months until Oct'07 constitute the observation period of our study.

As all of the applications were being used productively since early 2005 , the project has been in maintenance mode ever since. Typical tasks included: requirements elicitation, continuous forward development of new features and change requests, bug fixes, user support as well as deployment of new releases on the live servers

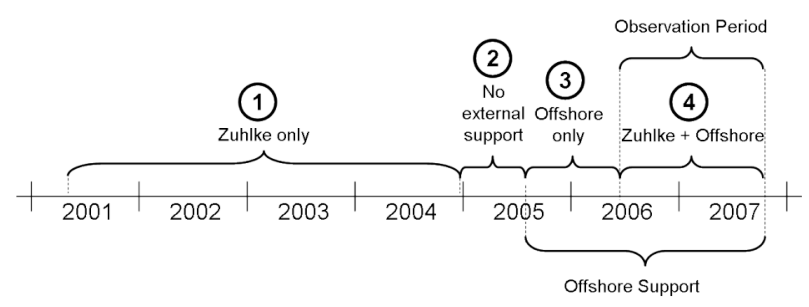

Figure 1. Course of the project, divided into four phases with varying external support.

including data migration. Furthermore, much effort was put into the improvement of product quality and development processes and the upgrade of development tools and infrastructure.

\section{Problems and Challenges}

When entering the project in Jul'07, it soon became clear that things were not running smoothly. However, it was difficult to identify all problems at once and find the reasons behind them; rather, symptoms were observed. Even after some time, still new problems were detected. In this section, we present a list of problems and challenges gathered along the observation period. The list is roughly sorted by scope, i.e., product, process, and people.

\subsection{Product}

Problems concerning the product were easiest to discover. They included:

- Misunderstanding of requirements. Forward development was at the time mostly performed by the offshore team. The delivered work packages were largely lacking in quality. Rather than the offshore team, the loose setup of the offshore project was probably the cause of the problem. Requirements were misunderstood which led to unusable work packages, which required rework.

- Missing offshore tests and wrong priorities. Testing was done, if at all, intuitively and manually in a very local context. Side effects on existing functionality were not considered. The findings were hardly reproducible, as no written protocols existed. Important engineering concepts like architectural styles or good coding principles were ignored, in sheer ignorance or due to wrong priorities. It seemed more important for the offshore team to implement new features and finish change requests quickly rather than producing high quality code. This led to hardly maintainable code, e.g., 
duplicated code and violated architectural principles, which required extra efforts to get it fixed again. Returning an item of rework to the offshore team always entailed a high communication effort, i.e., detailing the reasons for giving the item back and specifying how the results should look like.

- Further product-related problems. The applications to be maintained had reached a bad state, and quality was weak. The applications were buggy and partly instable. Communication with customers took a lot of time, in order to understand their needs and calm them down. However, the applications were not in a bad state from the beginning. Two to five years ago, the applications were written, tested and documented properly, as suggested by the data available from the versioning system. There was even a nightly build system available on the basis of scripts and cron jobs. Unfortunately, the tests and the builds were not running anymore. Finally, the used libraries and server software were rather old and not updated, and the computer infrastructure had simply become obsolete. This clearly underlines the decayed state of the applications. It seems that at a certain point of time, all good engineering principles were forgotten in favor of pure functional development. This must have happened one to one and a half years before our observation period.

\subsection{Process}

Problems in product quality are often caused by defects in the development process. What was going wrong in the process was detected only after a certain period of time and included:
- Unclear status of offshore work. After giving a work package offshore, there was no feedback until delivery, which was typically up to three weeks later. In the meantime, it was unclear how long it would take until delivery and to what extent the package was finished. These long feedback cycles made release planning almost impossible. This also affected the checkins to the software repository in that code was not submitted until it was thought to be completely finished. Consequently, check-in times took up to half a day, due to the size of the complete work packages and the speed of the connection to the offshore location.

- Missed milestones due to insufficient estimates. Estimates of work packages were made neither onshore nor offshore, and hence a cornerstone for release planning was missing. Rather, milestones were based on customer wishes. Who cried loudest got the next release date - independent of the content and the actual effort. These dates often could not be met, and some release dates had to be postponed several times. Obviously, customers were not happy at all and trying to increase pressure. Initially, there were promises to customers to deliver a certain functionality that went back up to one year. There was no application, which had no outstanding delivery delays.

- Further process-related problems. Process issues also affected the onshore site. Development did not follow any particular process. Work was done in an adhoc way, and so was release planning. And there was no control mechanism available to see how well or badly we were really working.

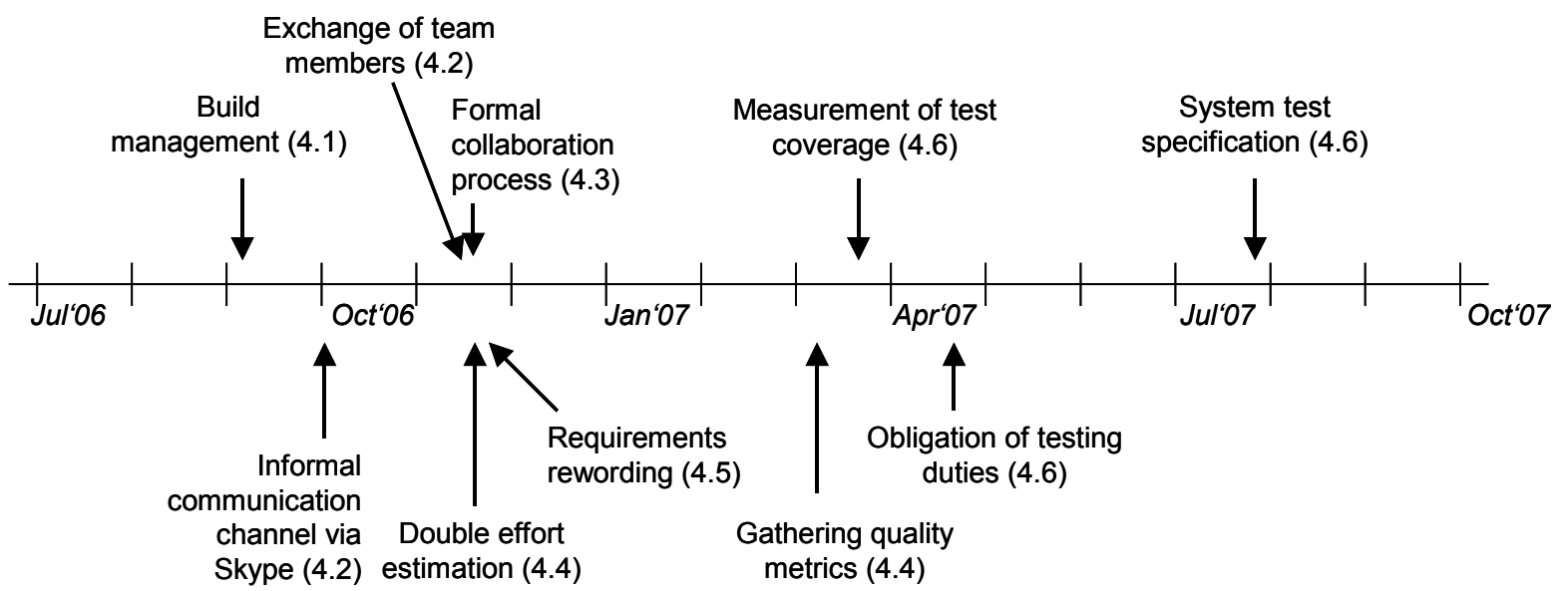

Figure 2. Overview of the measures taken in order of inception 


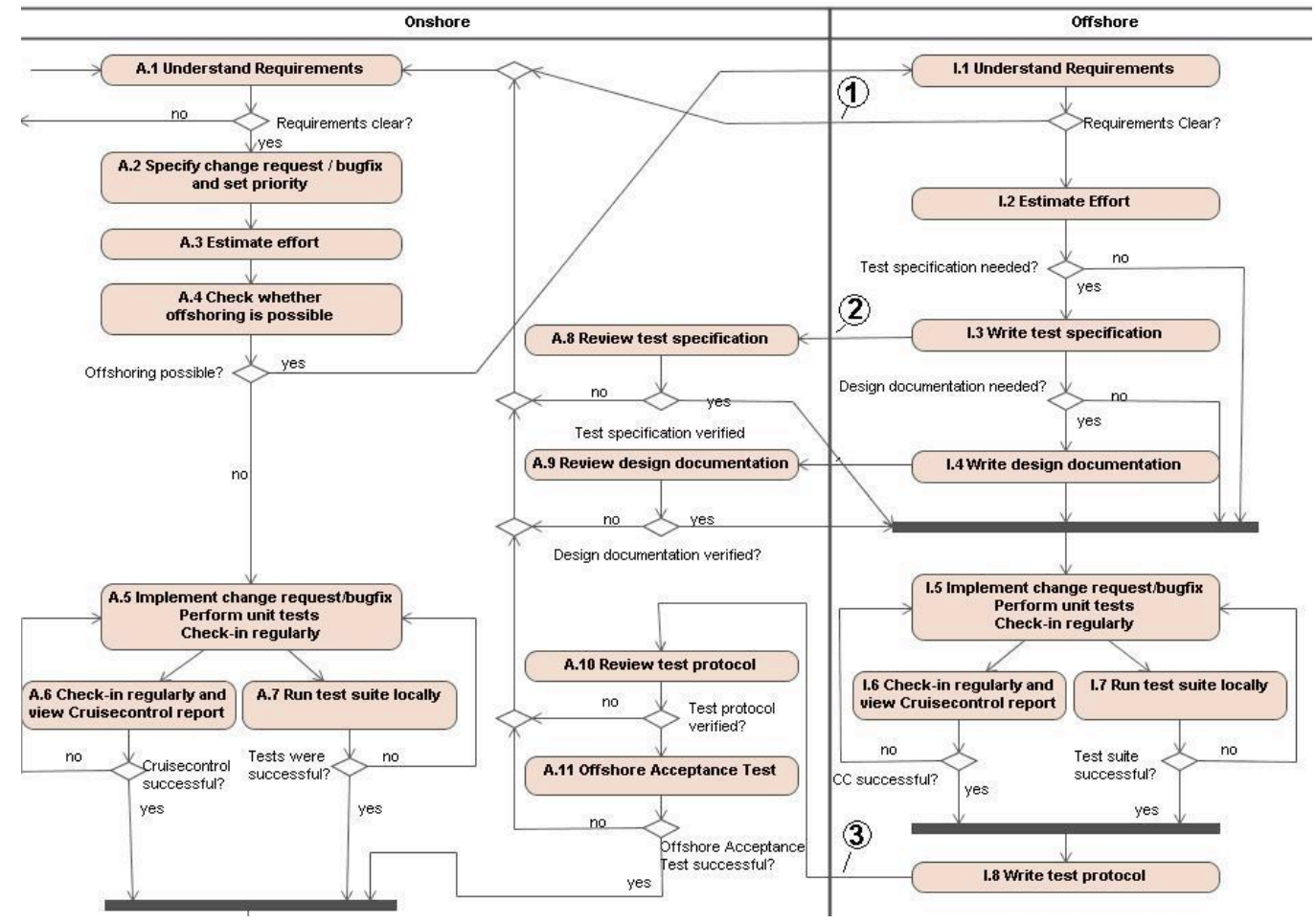

Figure 3. Onshore-Offshore collaboration process.

\subsection{People}

To make statements about people takes the longest observation time. It is not the intention to report about single individuals but about the offshore team as a whole. People problems included:

- Lack of identification. One of the first things we noticed is that apparently the identification with the project was related to the distance. In the beginning, we could not determine with the offshore colleagues a high sense of belonging to the project. Especially in situations, when something went wrong onshore that might have been caused by offshore work, the support by the offshore team was small. Instead, development as usual continued, and onshore problems were mostly ignored. The offshore team was working far away and rather isolated. It was difficult to transport the onshore atmosphere there, and probably they did not even want to get it.

- Insufficient knowledge of testing. Concerning programming, the offshore colleagues were all quite well trained. However, most of them were junior and had only little knowledge about testing, mostly limited to the basics of unit testing. Anyway, it was all the time difficult to get experienced senior team members.

- Further people-related problems. A further issue to be mentioned here is probably related to the two previous ones: Fluctuation. During the 16 months observa- tion period, three out of five offshore team members left the project and were replaced by new ones. This might be related to the missing identification with the project or to the development experience gained which made new positions possible. It is clear that each change implied a loss of knowledge. Search for new members and the initiation to the project took considerable time. In the observation period, our offshore partner relocated our team from Bangalore to Mysore, which is about three hours by train. Most of the team members did not want to move in the beginning. Finally, they all stayed but the onshore team had difficulties to convince them. For the project, exchanging even half of the people at the same time would have meant a long deadlock.

\section{Measures Taken}

Facing the problems discussed in Section 3, a number of measures were taken. In the project, not all problems were actually related to the offshore context, nor were the measures purely offshore specific. However, in this paper we clearly focus on offshore-related problems and measures. The major measures taken are listed in Figure 2 in chronological order of inception (and are written in italics in the text below). Their implementation naturally took weeks, let alone months, or is even still ongoing. 


\subsection{Build Management}

The first measure introduced was to establish build management (see Figure 2) and set up a continuous integration environment [5]. We decided to use the popular tool CruiseControl [12] as it fits well into our development environment for Java and Ant. As the nightly builds were not running anymore, it was probably even less effort to switch to a modern tool than to reactivate the existing shell scripts and cronjobs.

Setting up a common build environment entailed the following three benefits: (A) It defines a common way of building a product, independently of the team members' local settings. On-/offshore discrepancies due to different build procedures could easily be eliminated. (B) Notifications on changes in the code are distributed faster as each build result is sent to every team member by e-mail. This reduced the need to communicate changes and allowed earlier detection of potential conflicts when two people were working on the same code parts. Moreover, you get feedbacks from people working at other locations. In a distributed setup, this can replace to some extent the informal communication facilitated by a joint office. (C) It eases communication when working on a specific issue. As CruiseControl splits the continuous flow of code changes into transparent and defined sets, and provides as labels discrete build numbers, any set of changes can easily be referred to. This greatly reduced misunderstanding in our project.

\subsection{Communication}

As stated above, communication was not running smoothly in the beginning. As communication is the basis for many tasks in a software project, e.g., assigning tasks, status reporting, clarifying misunderstandings, etc., weak communication will worsen existing problems [6]. Beyond the established communication channels phone calls, e-mail, and Bugzilla [13], we introduced new ones:

- Most importantly, as it turned out in hindsight, was the introduction of an informal communication channel (see Figure 2). We decided to use the chat functionality of Skype ${ }^{1}$. This had several positive effects. A) The barrier to communicate was drastically reduced in comparison to phone calls. Communication could take place semi-synchronously, i.e., principally synchronously, yet by tolerating more delays than a phone call would do. Communication takes place off-the-record and would not be evaluated or recorded. B) As the

\footnotetext{
${ }^{1}$ Skype was chosen for technical reasons as it gets across most firewalls. Other products might have revealed a similar effect.
}

presence status of every team member is always visible, a better team feeling grew up. Chatting supported the exchange of friendly gestures. The two locations came much closer to each other. This is especially useful if there is only a small overlap in working times due to time zones. C) At a technical level, the exchange of artifacts and data was eased as most chatting systems allow sending files to the chat partners. Besides all advantages, one has to say that Skype is not suitable for any official communication as the recording capabilities are quite bad. If a discussion yields any important results, these must be logged somewhere else, e.g., in the corresponding Bugzilla entry.

- A similarly high impact on our communication behavior resulted from the exchange of team members between onshore-offshore locations (see Figure 2). Three offshore team members so far - one at a time visited Switzerland and were working at the customer's location for about 1 to 4 months each. Furthermore, an onshore member once went to India for 4 weeks. Again, we could benefit from several advantages, the two major ones concerning communication. A) Having an offshore team member on-site channeled most communication to the offshore team over this team member. Complex matters were intensively discussed from face to face, before the results were transferred in a condensed form to the offshore team by the on-site offshore team member. To do this, phone calls as well as chats were the favorite media. The communication was then much efficient, due to linguistic and personal closeness of the offshore team members amongst each other. The mission of the onshore team member at the offshore location was to inspect and improve the working environment and to give trainings. B) Another important advantage of the on-site presence of the offshore team member is to hold the offshore team more accountable on what they are doing. Instead of observing reactions on their produced work from a distance, the local offshore member is able to experience closely the going live of a new release and to feel responsible if customer reactions - positive as well as negative ones - are triggered by offshore work. Eventually, the onshore atmosphere was transmitted easily to the rest of the offshore team.

- Another useful communication channel introduced was Microsoft Netmeeting allowing sharing one's desktop. Complex situations are easily explained by showing an example rather than providing long texts. In some rare cases this medium proved to be quite useful and saved us a lot of time.

- A mailing list was introduced that allowed everybody to send a message to all other team members. This list was mainly used for announcements of major events or releases. However, the envisioned technical discussions did not take place here but were carried out 
via chat. The actual benefit of the mailing list was small.

- Concerning the existing communication channels phone conference and Bugzilla, the frequency and content did not change much. However, communication via e-mail strongly decreased, and was mostly replaced by chatting. The strong increase in overall communication shows that there was a pressing need to exchange information that could not be satisfied by the few existing channels in the beginning.

\subsection{Collaboration Process}

In principle, collaboration among on- and offshore locations improved already with the introduction of the informal communication channel. Still, the collaboration process was not satisfactory, leading regularly to an unclear status of work items, e.g., whether an item had already been started or not. We therefore decided to introduce a formal collaboration process (see Figure 2) as a common framework and apply it pragmatically [10]. The process allowed us to better express expectations and to reduce communication effort (see Figure 3 ). The figure is cut in order to show the on-/offshore collaboration and is not completely displayed (activities related to the application users, i.e., requirements elicitation and acceptance tests, are hidden). This process description contains for each work item given offshore three major feedback cycles. Initial feedback is obtained after the specification of the new work item has been received offshore. The colleagues send back the open questions concerning the specification or an "Everything understood" signal (1). Later on, a "requirements rewording" was introduced whose details are described in Section 4.5 below. A second feedback constitutes an estimate of the effort and time to finish (2), as an input for planning upcoming releases (see also Section 4.4 below). Finally, together with the finished code, we receive the test protocol showing that the test cases were indeed executed (3). In the textual descriptions of the activities, it is specified when and how Bugzilla states have to be changed. On the one hand, progress can be observed without communication overhead. On the other hand, the risk of misunderstandings is reduced with each feedback cycle.

\subsection{Project Management}

Concerning project management there were two major measures that affected both onshore and offshore development:

To get a better overview about offshore activities a socalled task tracking sheet was introduced. This sheet gathers cumulatively all open, on-going, and closed offshore work packages. An updated sheet is sent once per week by e-mail. Usually, a work package is related to a Bugzilla entry. For each work package, expected start date, effort, completion date, and assignee are given. As soon as a work package is in the works, actual start date and spent effort are added. A detailed history to each work package documents any changes of the estimates, for example due to changed requirements or detected bugs. With this measure, we got a much better overview of which tasks were in the works and about progress, and it allowed us to schedule release dates based on the work packages to be included. Furthermore, it probably helped raising project identification, as we showed a great interest in the offshore team's work. Estimates once given by the offshore team were in the very most cases met except circumstances changed considerably. Our colleagues were indeed keen to fulfill their own promises. With these offshore estimates available, we were able to introduce a measure we call double effort estimation (see Figure 2 ). Before giving work packages offshore, we made our own rough estimates of the required effort without telling them. After receiving their first estimates, we could compare them to ours. We did this not to blame them, but to get an impression whether they understood our requirements right. If their effort was more than three times higher or lower or more than five days greater or smaller, we asked back how they got these numbers. In this way, we could clarify misunderstandings at an early stage. As this measure means quite a large effort on our side, we were using it only for a few months in a phase when release planning was difficult [8]. Later on, we stopped it in favor of the requirements rewording described in the next section, which is less useful for scheduling but more effective to detect misunderstandings.

One of the last major measures was the introduction of gathering quality metrics (see Figure 2). On the one hand, test coverage was taken to document the progress of fixing old tests and writing new ones. To show the importance besides functional development, quarterly targets were fixed, and time slots were reserved expressly for writing new test cases and fixing old ones. On the other hand, the states of work packages in Bugzilla where taken to get an impression on quality. We distinguished between customer and internal requests, and between bugs and change requests. Customer requests were rated higher than internal ones, and bugs higher than change requests. We observed how many work packages had to be re-opened. Reopening due to quality reasons was rated high, and low, in case of changed requirements. And we measured when it actually was re-opened. Re-opening before delivery was rated low, after delivery high. In total, the worst case that could happen in our quality scale was the re-opening of a work package describing 
a bug reported by the customer due to quality reasons after the fix was actually delivered, i.e., the bug was actually not fixed correctly and the customer was aware of it. To further improve quality of the offshore cooperation, a most recent action was to start a Six Sigma project at Green Belt Level. Metrics have already been defined to determine the current situation and identify potential for further improvements.

\subsection{Requirements Management}

As misunderstandings of requirements were often the cause for rejected work package deliveries, we aimed to reduce the risk of getting the wrong thing implemented. The problem with a rejection of a work package is not only that it is not available at the expected time and requires additional implementation effort, but given an offshore development setup, it requires additional onshore effort 1) for explaining what exactly was wrong and eventually 2) running another acceptance test. Beyond the extra effort incurred, for both sides the motivation decreases which can affect overall quality.

The measure taken is called requirements rewording (see Figure 2), and the idea is to get feedback on the correctness of the requirements before implementation is started. Nobody loses face if requirements are corrected in the beginning. Our initial work package descriptions used to be rather short and quite imprecise. This made phone calls and chats necessary, yet the discussion results were often not documented. As a first step, we improved the situation by writing more detailed work package descriptions, trying to be more precise and adding screenshots and mockups wherever possible $[1,3]$. We started to write down oral agreements. As a second step, the actual rewording was introduced. We expected every work package to be reformulated by the offshore team in their words. Moreover, they had to provide additional screenshots and a test specification consisting of several test cases (for manual execution) with input parameters and expected results to test the new functionality. This reformulated and extended requirements document was sent back to have us agree on it. Using this procedure, we had much higher confidence that our work package description had been understood correctly. If we got the impression of a misunderstanding, we tried to make our description more precise or took the issue up for discussion. As a side effect, the specified test cases were the basis for the test protocol that we expected, once implementation was finished. These test cases covered the new functionality pretty well. However, they were typically limited in scope. Thus, we could not prevent unexpected side effects with this measure, and therefore decided to extend our testing strategy.

\subsection{Testing Strategy}

Testing was an important topic in the project right from the beginning. We could observe this from the many pre-existing test artifacts. For most applications, we found both automated test classes as well as test specifications for manual system tests. However, they were not properly maintained and in bad state at the start of the observation period. Three major measures were taken to remedy the situation:

To start with, we decided to execute all existing test classes again on a regular basis, i.e., we included them into the automated builds. In total, there were over 800 test cases available, one third of it failing. It soon became clear that we would not be able to fix this huge amount with a single concerted effort. But we decided to continuously spend little effort to fix test cases and write new ones besides normal development with a special focus from time to time. Anyway, the generated reports from the build server helped us to keep this task in mind and also to track our improvements with an increasing number of passing tests. Until today, less than every fourth test case is failing. Counting the number of tests was for us not satisfactory enough to determine the quality of our tests. Therefore, we opted, as a first measure, for the measurement of test coverage (see Figure 2), and adopted Clover [4] to track the improvements of different coverage values. Not surprisingly, statement coverage was below $20 \%$ in the beginning. Until today, we could raise this number to more than $25 \%$ by fixing test cases. In the beginning, testing was entirely an onshore responsibility, yet the effort largely exceeded our onshore capacity. As testing was not common on the offshore side, we started to create awareness and emphasize the importance of testing, declaring, as a second measure, an obligation of testing duties (see Figure 2). We gave trainings for writing unit tests and interpreting test coverage data. And finally, we were able to distribute quarterly targets to further increase coverage.

As the benefit of automated tests lies in the long term [2], their introduction had only little effect on our current quality problems. To harness quality problems in new work packages, we established a three-stage approach. Firstly, we carried out a manual acceptance test for each work package delivered by offshore, including the inspection of the offshore test protocol. Secondly, we integrated all changes in our test environment and executed additional intuitive and ad-hoc tests. For each release, we needed two to ten release candidates to integrate all changes and reach an acceptable stability. Thirdly, we asked our customers to perform their acceptance tests on our mature release candidates. However, it turned out that though many bugs were discov- 
ered using this method, still too many bugs occurred after live release (mainly discovered by customers). The only way to significantly improve quality was to switch from intuitive to systematic testing and again to involve our offshore team. We wrote a complete system test specification (see Figure 2) including basic functionality as well as tests for all newly occurring problems. Furthermore, we started adding existing test cases from the work package descriptions to the system test specification. The participation of the offshore team was twofold. We asked them to perform a system test on the offshore site as well. And we taught them to write themselves new test cases for the system test. With our combined effort, we managed to release an application at the end of the observation period for which no customer complaints have been reported so far.

\section{Impact}

In this section, we aim to assess the impact of the measures taken (Section 4) on the problems under consideration (Section 3). How can we be sure that our measures had any effect at all? Which measures are suited to solve which problems? Which measures had most impact at all? We are sharing our observations in this section qualitatively, and substantiate some of them quantitatively with numbers gathered from the project context.

\subsection{Impact on Communication}

To get an impression on the effect of the introduced measures concerning communication, we evaluated how intensively different communication channels had been used at the beginning and at the end of the observation period (see tables 1 and 2). We counted e-mails, phone calls, phone conferences, and Bugzilla entries, and observed and interviewed team members on- and offshore concerning their face-to-face communication. Out of these data, we distilled a qualitative intensity scale: "_" (rarely used), "o" (averagely used), "+" (strongly used), and "++" (heavily used). We further distinguished who was communicating with whom and derived the following cases: communication solely among onshore ("On-On") and offshore team members ("Off-Off"), communication from on- to offshore location and vice versa ("On-Off"), and communication among the one exchanged offshore team member while located onshore and his/her colleagues located offshore ("Off@On-Off"). Comparing the two tables reveals two interesting observations:

Firstly, having a look at Table 1, one can see that team members located at the same site heavily communicated face to face, however between the two sites only average communication took place, mainly over phone conference or indirectly using Bugzilla. In Table 2, the newly introduced channel "Chat" (new row "Chat") was intensively used mainly for communication between the two sites, whereas the existing channels did not change their usage levels. This suggests that with the introduction of Skype an urgent need for informal communication could be satisfied, yet that the existing channels still had their necessity.

The second observation concerns the columns. In Table 1 , the column representing the communication between the two locations ("On-Off") is rather pale, meaning the intensity over all channels is rather low. With the one offshore team member located onsite, a strong increase in phone and chat communication between this member and his/her team could be observed (new column “Off@On-Off”) which was the very intent of relocation. So, the measure "having one offshore team member working onsite" indeed overcame a bottleneck in communication between the two locations.
Table 1. Communication intensity grouped by channel at the beginning of the observation period.

\begin{tabular}{|r|c|c|c|}
\hline Channel & On - On & On - Off & Off - Off \\
\hline Face to Face & ++ & n.a. & ++ \\
\hline Phone Call & n.a. & - & n.a. \\
\hline Phone Conf & n.a. & o & n.a. \\
\hline Bugzilla & o & o & o \\
\hline
\end{tabular}

Table 2. Communication intensity grouped by channel at the end of the observation period.

\begin{tabular}{|r|c|c|c|c|}
\hline Channel & On - On & On - Off & Off - Off & Off@On - Off \\
\hline Face to Face & ++ & n.a. & ++ & n.a. \\
\hline Phone Call & n.a. & - & n.a. & ++ \\
\hline Phone Conf & n.a. & 0 & n.a. & 0 \\
\hline Bugzilla & o & + & 0 & - \\
\hline Chat & - & + & 0 & ++ \\
\hline
\end{tabular}




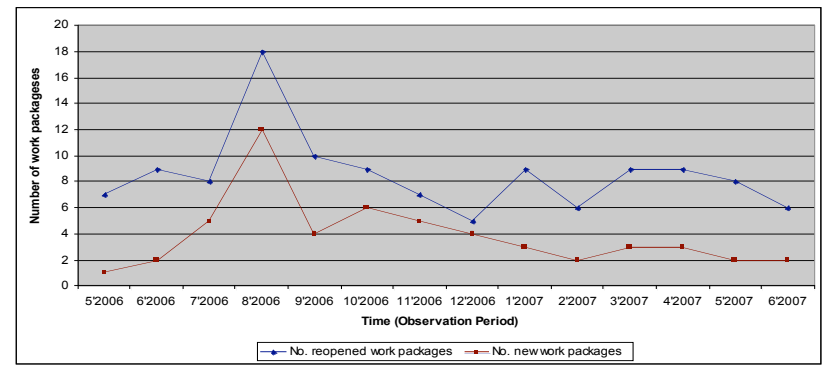

Figure 4. Re-opened and new work packages.

\subsection{Quality of Work Packages}

Many measures targeted, directly or indirectly, the problem of rather weak quality of work packages, e.g., requirements rewording, more feedback cycles in the collaboration process, as well as most testing measures. As quality is not directly measurable, we decided to observe Bugzilla entries to obtain an impression of quality. The more new work packages have to be opened, and even more importantly, the more closed work packages have to be re-opened, the more problems have generally been detected. In Figure 4, the curves of the two metrics are given for the observation period. The quite high peak around August'08 was the beginning of manual testing which revealed a lot of problems. However, in the following months, even stronger and more systematic testing was applied (see Section 4.6), yet both curves decreased to a more acceptable level. Still, there is room for improvement. But in contrast to the beginning, we reached a much better level of quality.

\subsection{Effectiveness of Measures}

In Table 3, we have compiled our experience with the major measures introduced during the observation period. The rows refer to the major problems related to the offshore context, as detailed in Section 3. The columns represent the major measures discussed in Section 4. The cells show how well a measure was contributing to the solution of a particular problem: "++" (heavily), "+" (strongly), and " 0 " (scarcely). Each measure typically affected several problems. For qualitatively comparing the usefulness of individual measures, we counted for each measure the number of "+" values in the list of problems. These totals can be found in the last row. Surprisingly, two measures are topping all others by far: the introduction of an informal communication channel and the exchange of team members. These two measures contributed to the solution of all problems listed, which agrees with our personal intuition and the feedback of the teams. This underlines the key role of personal communication, also in software maintenance. A high number of "+" values may be an indicator of the importance of individual measures, yet only the combination of several measures allows tackling the problems properly.

\section{Lessons Learned}

The presented measures have led our software maintenance project, which is "locked" in the "offshore reality" to success. We think that these measures not only apply to similarly locked maintenance projects, but even to software maintenance projects at large and

Table 3. Problems - Measures Matrix.

\begin{tabular}{|c|c|c|c|c|c|c|c|c|c|c|}
\hline Problems: & 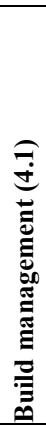 & 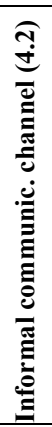 & 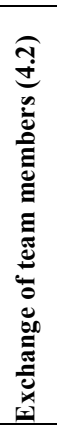 & 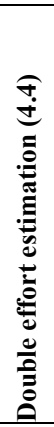 & 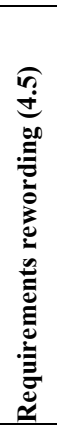 & 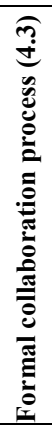 & 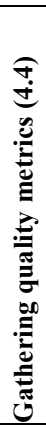 & 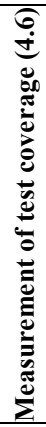 & 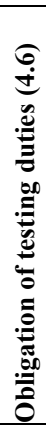 & 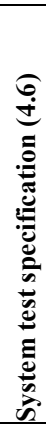 \\
\hline Misunderstanding of requirements (3.1) & 0 & ++ & ++ & + & ++ & + & + & 0 & + & 0 \\
\hline Missing offshore tests and wrong priorities (3.1) & + & + & + & + & 0 & + & + & + & ++ & ++ \\
\hline Unclear status of offshore work (3.2) & + & + & ++ & + & + & ++ & 0 & 0 & 0 & 0 \\
\hline Missed milestones due to insufficient estimates (3.2) & + & + & ++ & ++ & + & + & 0 & 0 & 0 & 0 \\
\hline Lack of identification (3.3) & + & ++ & ++ & 0 & 0 & 0 & 0 & 0 & 0 & 0 \\
\hline Insufficient knowledge of testing (3.3) & + & + & ++ & 0 & + & 0 & + & ++ & ++ & ++ \\
\hline Total (number of + signs) & 5 & 8 & 11 & 5 & 5 & 5 & 3 & 3 & 5 & 5 \\
\hline
\end{tabular}


hence beyond the offshore context.

- Build Management: Is an essential means to easily integrate all kind of automation, provide fast feedback to developers and trace back side effects to a small set of changes.

- Communication: The necessity of communication correlates with the number and size of work packages. Many small tasks require more communication effort than fewer larger ones. Communication among distributed locations might improve using several different communication channels.

- Collaboration Process: The more distributed, the more process might be required. A well-defined process helps to follow up on the other locations' current state and to improve cooperation while reducing the effort necessary for communication.

- Project Management: As with management by phone, it is always difficult to get an exact picture of a distant team. Additional means are important to get status information and detect deviations early. Especially effort and quality are worth to be continuously monitored.

- Requirements Management: Having precise, complete und understandable requirements is even more relevant for distributed locations and varying cultures. Potential for misunderstanding is large. Well-understood requirements reduce the risk of expensive extra loops with the offshore team.

- Testing Strategy: For testing, it is especially useful to delegate as much testing as possible to the developing locations to keep communication between locations small. Nevertheless, each work package should be tested for acceptance onshore.

\section{Conclusion}

We did not discuss yet whether it is useful to apply offshoring in maintenance projects. This project was locked in the "offshore reality", that is, the requirement to develop and maintain mostly offshore was a given and unquestionable. Whether offshore development makes sense or not depends on various factors, but in our opinion mainly on the size of the work packages and the communication needs. Eventually, it is the criteria cost, time to market, and quality that will decide over success. To benefit from smaller wages, extra effort for communication and trainings must remain low. This may become true only if the teams work well together. Time to market for medium to small work packages can hardly be beaten with an offshore development. Product quality mainly depends on priorities and the available developers. Whereas offshore developers are not in short supply, it is still difficult to find highly qualified professionals.
This suggests that software maintenance projects with short release cycles and small work packages are not well suited for offshore development. However, our study shows that the bundle of measures presented, coupled with their disciplined implementation, makes successful software maintenance projects in the offshore reality a possibility.

\section{References}

[1] Antoniol, G. and Guéhéneuc, Y.. Feature Identification. A Novel Approach and a Case Study. In Proceedings of the International Conference on Software Maintenance (ICSM'05), pages 357-366, Budapest, Hungary, September 2005. IEEE.

[2] Berner, St., Weber, R., and Keller, R.K.. Enhancing Software Testing by Judicious Use of Code Coverage Information. ICSE 2007, pages 612-620.

[3] Cheng, B. and J. Atlee. Research Directions in Requirements Engineering, in Future of Software Engineering 2007, pages 285-303, L. Briand and A. Wolf, Editors. 2007, IEEE.

[4] Clover, www.cenqua.com/clover.

[5] Fowler, M. Continuous Integration. martinfowler.com/ articles/continuousIntegration.html

[6] Herbsleb, J. Global Software Engineering: The Future of Socio-technical Coordination, in Future of Software Engineering 2007, pages 188-198, L. Briand and A. Wolf, Editors. 2007, IEEE.

[7] Niccolai, J. Gartner. Five reasons why offshore deals go bust, 2005, www.computerworld.com/managementtopics/outsourcing/story/0,10801,102677,00.html.

[8] Sneed, H. and Huang, S.. Sizing Maintenance Tasks for Web Applications. In Proceedings of the 11th European Conference on Software Maintenance and Reengineering (CSMR-2007), pages 171-180, Amsterdam, The Netherlands, March 2007. IEEE.

[9] Tiwana, A. Beyond the Black Box: Knowledge Overlaps in Software Outsourcing, IEEE Software, 21(5), Sept. / Oct. 2004, pages 51-58.

[10] Weber, R., Helfenberger, T., and Keller, R. K., Fit for Change: Steps towards Effective Software Maintenance, Proceedings of the International Conference on Software Maintenance (ICSM'05), p. 26-33, Budapest, 2005.

[11] Wichmann, K.P and Keller, R. K. OffshoreMöglichkeiten besser genutzt: Assessments zur Strategie, Partnerwahl und Durchführung. OBJEKTspektrum, 14(5):55-59, Sept. / Oct. 2007. SIGS Publications. In German; Better use of offshore opportunities: assessments of strategy, partner selection, and operations.

[12] CruiseControl, http://cruisecontrol.sourceforge.net.

[13] Bugzilla, http://www.bugzilla.org. 